\title{
Montag ist Stresstag fürs Herz
}

\section{Hier steht eine Anzeige.}

Der Montag ist aus kardiologischer Sicht ein schwarzer Tag, weil sich hier laut einer kanadischen Langzeitstudie unerwartete kardial bedingte Todesfälle häufen.

Den Ergebnissen einer Nachauswertung der kanadischen Kohortenstudie Manitoba Follow-up-Study (MFUS) nach ereignet sich der unerwartete plötzliche Herztod mit einer Rate von $21 \%$ anteilig am häufigsten an Montagen.

Bemerkenswerterweise war das Risiko, einen unerwarteten plötzlichen Herztod zu erleiden, bei jüngeren Männern am höchsten. Bei der Suche nach Erklärungen stießen die kanadischen Forscher auf zwei Studien aus Litauen: Deren Autoren hatten 2001 herausgefunden, dass die Häufung plötzlicher Todesfälle zu Wochenbeginn wahrscheinlich mit Alkoholexzessen am Wochenende zusammenhing. Auch für die kanadischen Forscher scheint dieser Zusammenhang plausibel.

Tate RB, St John PD. Am J Epidemiology, online 13. November 2017;

https://doi.org/10.1093/aje/kw×343

Androgenetische Alopezie

\section{Haarfülle zeigt Prostatakrebsrisiko}

\section{Anhand der Haarpracht eines Mannes lässt sich ver-} mutlich sein Prostatakarzinomrisiko abschätzen.

Ergebnisse einer chinesischen Metaanalyse deuten auf einen Zusammenhang zwischen der androgenetischen Alopezie und Erkrankungen der Prostata. Mit einer androgenetischen Alopezie stieg das Risiko, an einer aggressiven Form des Prostatakarzinoms (Odds-Ratio [OR] 1,60, 95\%-Konfidenzintervall [KI] 1,37-1,86) oder einer benignen Prostatahyperplasie (OR 1,26, 95\%-KI 1,05-1,51) zu erkranken. Mit nichtaggressiven Formen des Prostatakarzinoms hingegen war die androgenetische Alopezie (OR 1,05, 95\%-KI 0,90-1,06) nicht assoziiert.

Dass der Verlust der Haare als Indiz für ein erhöhtes Risiko für Prostataerkrankungen gelten kann, führen die Urologen auf ähnliche pathophysiologische Muster zurück, in denen Androgene und das Alter eine Rolle spielen. Belastbare Beweise gäbe es für diese Hypothese aber nicht, wie die Urologen betonen. 